e-migrinter

\title{
e-Migrinter
}

$21 \mid 2020$

Famille et migrations

\section{Mariées mais séparées}

Migration sénégalaise en Catalogne depuis une perspective féminine

Marème Niang-Ndiaye

\section{(2) OpenEdition}

Journals

Édition électronique

URL : https://journals.openedition.org/e-migrinter/2513

DOI : $10.4000 /$ e-migrinter.2513

ISSN : 1961-9685

Éditeur

UMR 7301 - Migrinter

Référence électronique

Marème Niang-Ndiaye, " Mariées mais séparées », e-Migrinter [En ligne], 21 | 2020, mis en ligne le 15 janvier 2021, consulté le 20 mai 2021. URL : http://journals.openedition.org/e-migrinter/2513 ; DOI: https://doi.org/10.4000/e-migrinter.2513

Ce document a été généré automatiquement le 20 mai 2021.

Tous droits réservés 


\title{
Mariées mais séparées
}

Migration sénégalaise en Catalogne depuis une perspective féminine

\author{
Marème Niang-Ndiaye
}

\section{Introduction}

1 Les mariages à distance sont devenus une réalité courante dans la société sénégalaise. Leur développement ces dernières années est en grande partie corrélé aux mutations qu'a connu la migration internationale des Sénégalais vers l'Europe. En effet, à partir des années 1990, le durcissement des conditions d'entrée et de séjour dans nombre de pays de destination des Sénégalais (France, Belgique, Allemagne, Italie, Espagne), la fermeture et les restrictions de circulation de migrants, les conditions complexifiées de regroupement familial et l'accès difficile à un marché du travail européen soumis à des réglementations strictes, ont constitué des facteurs structurants de l'existence de ces mariages en contexte sénégalais.

2 Documenté depuis plus d'une vingtaine d'années en sciences sociales, le mariage à distance a fait l'objet de nombreux travaux qui se sont intéressés aux différents enjeux liés aux arbitrages et au maintien des liens matrimoniaux par-delà les frontières. Les approches sociologiques ont été les premières à théoriser les possibles conséquences du départ d'un des conjoints sur celui qui reste. Des situations de tension et de déstabilisation des liens du mariage en contexte migratoire ont été étudiées (Jolly et Reeves, 2005 ; Fouron et Schiller, 2001 ; Mahler, 2001 ; Glick, 2010 ; Caarls et Mazzucato, 2015 pour ne citer qu'eux). D'autres analyses ont mis l'accent sur les positions et pouvoirs acquis par les femmes grâce à la migration et les répercussions sur le couple. Ainsi, si la migration des hommes ouvre une marge de contestation et de liberté aux femmes, elles les propulsent souvent comme principales contributrices au maintien du foyer. Ces situations engendrent souvent une déstabilisation de certains systèmes socioculturels où les rapports entre les sexes s'en trouvent fortement remis en cause (Hirsch, 2003 ; Levitt, 1998 ; Zontini, 2003).

3 Les effets des politiques migratoires sur les couples «transnationaux » ont également fait l'objet de recherches quantitatives poussées. Elles montrent que si la vie 
transnationale émane souvent d'une décision choisie au sein du couple, elle n'est pas toujours effective, ni réalisable, du fait des procédures de réunification familiale parfois drastiques et draconiennes (Baizan et al., 2011 ; Mazzucato et Schans, 2011).

4 Si ces analyses fécondes, sans être exhaustives, ont permis de comprendre l'importance des approches transnationales de la migration dans la compréhension des rapports entre les sexes et les tensions inhérentes à la distance et la séparation au sein des couples " transnationaux », les enjeux liés au vécu, aux pratiques et représentations des femmes mariées, qu'elles « restent » dans le pays d'origine et/ou qu'elles « partent » en migration, constituent le parent pauvre de ces études.

5 À partir d'une approche par la géographie sociale, cette contribution se propose de poser le débat sur les tensions qui existent entre le mariage à distance des Sénégalais et Sénégalaises et les mutations sociales et sociétales engendrées par la migration internationale. L'entrée par les pratiques et perceptions de femmes mariées permettra de répondre à deux principales questions. Premièrement, quel est l'impact de l'absence prolongée des hommes au sein de leur famille/couple sur la place et le rôle des épouses, et comment peut-on y voir, à une échelle plus globale, une mutation des rapports sociaux de genre au Sénégal ? Deuxièmement, dans quelle mesure les " effets de liens " (ici les héritages socioculturels, les normes et traditions sociales) influent sur les arbitrages matrimoniaux surtout lorsque la migration concerne la femme mariée ? Une démarche «multisituée» qui s'appuie sur le Sénégal et sur la RMB (Région Métropolitaine de Barcelone), ${ }^{1}$ permettra de croiser différents discours, médiatiques et issus d'entretiens, pour tenter de répondre à nos objectifs de recherche.

\section{Quelles méthodes et données pour étudier la vie des couples séparés par la migration?}

Pour mener à bien cette recherche, nous nous somme appuyée d'une part sur les travaux d'une recherche doctorale (2010 et 2014) qui porte sur l'habiter en migration des Sénégalais et Sénégalaises, ainsi que des Gambiens et Gambiennes à Barcelone, et d'autre part, sur le recueil de témoignages de femmes à partir de sources médiatiques. Le matériau empirique de la recherche doctorale concerne une série d'entretiens de type biographique auprès de 30 Sénégalais ( 17 hommes et 13 femmes) et de 15 Gambiens ( 12 hommes et 3 femmes) ${ }^{2}$, suivie d'une observation participante des lieux de l'habiter. L'enquête s'est déroulée dans 17 communes de la RMB où ces groupes étaient les plus importants en 2011 : Villassar de Mar, Teai, Callella, Badalona, El Masnou, Las Franqueses del Vallès, Hopitalet de Llobregat, Barcelone, Premia de Mar, Mataro, Pineda de Mar, Granolles, Canovelles, Cerdanyola, Terassa, Sabadell, Malgrat de Mar. Pour cette étude, nous avons choisi de nous focaliser sur quelques migrants et migrantes, tous et toutes avec un mariage en cours, et ayant plus de 18 ans. Ils et elles ont au moins un enfant et un conjoint ou une conjointe au Sénégal. Très peu de variations en termes de classe ou de niveau de vie sont observées dans ce groupe. Les données sur la dimension familiale ont été recueillies au cours de l'analyse de leur trajectoire de vie, à partir de questions relatives à leur situation familiale, aux rapports qu'ils établissent avec leurs conjoints (époux et épouses) et avec la famille élargie et de manière générale avec la société d'origine. Cette dimension familiale a fait ressortir différentes pratiques liées au don et au contre-don qui existent au sein du réseau familial et la manière dont ces pratiques transnationales se répercutent dans les 
arbitrages matrimoniaux mais aussi dans les rapports de ces migrants et migrantes au pays d'accueil (insertion professionnelle, sociale, perception, ancrage ou non ancrage). Ceci a permis de comprendre la complexité des liens qu'ils et elles tissent avec les différents espaces habités mais aussi les éventuels ajustements, négociations, tensions, effritements et souffrances qui accompagnent l'expérience migratoire et rythment les jeux d'intérêts, d'autorité et parfois de domination qui caractérisent les rapports de matrimoniaux.

7 Un intérêt particulier a été accordé aux sources médiatiques. Cela s'explique par le fait que le sujet étudié s'inscrit dans des problématiques sociétales d'actualité relayées dans l'opinion publique sénégalaise et qui se retrouvent à toutes les échelles de la vie sociale. Dans ce cadre, ces sources constituent une forme de " miroir » de la société sénégalaise à un moment $t$, permettant d'avoir une vision d'ensemble sur ces mariages à distance. Les témoignages de femmes d'émigrés étudiés sont issus de deux articles de presse du site Leral.net ${ }^{3}$ et du magazine en ligne ''Obs $^{4}$, et d'une émission télévisée appelée WAREEF diffusée du 03 au 09 février 2015 sur la TFM (Télévision Futurs Médias) ${ }^{5}$. L'exploitation et l'analyse distanciée de ces sources médiatiques a permis de mettre en lumière d'une part, l'acuité des problématiques que soulèvent ces unions au Sénégal et d'autre part, la prégnance de certaines réalités socioculturelles et contraintes qui pèsent sur la vie des couples séparés par la migration et sur leurs familles.

\section{Migration et mariage au Sénégal}

Depuis les années 1990, des études portant sur les couples « transnationaux » sénégalais ont permis de mettre en relief le rôle prépondérant de l'appartenance sociale et identitaire dans les arbitrages matrimoniaux (Mondain, 2009; Antoine et Dial, 2005 ; Mondain et al, 2012). Elles ont montré que les mariages à distance relèvent à la fois d'attentes différenciées dans les rapports entre conjoints mais aussi au sein de l'unité familiale (Mondain et al, 2012). En effet, dans la société sénégalaise, les relations matrimoniales restent généralement codifiées par des normes sociales et religieuses (religion musulmane, culture et tradition wolof) qui confèrent une certaine prédominance de l'homme sur la femme (Bara-Diop, 1985 ; Dial, 2008). Se faisant, quand l'homme migre, les règles de patrilocalité exigent souvent à la femme qui « reste »de co-résider avec le reste du ménage et dans certains cas avec la famille élargie (Antoine, 2002; Antoine et Dial, 2005; Mondain, 2009). Des tensions liées non seulement à la cohabitation avec la belle famille, aux rumeurs et commérages et mais aussi à la surveillance de la part des autres membres, constituent des épreuves qui rythment la vie de ces femmes (Mondain et al, 2012). Des situations de précarité autour de l'accès aux ressources qui marquent une certaine dépendance des femmes à l'égard de leur mari lorsqu'il s'agit de la « dépense quotidienne » ont aussi été soulignées (Mondain et al, 2012).

9 Parallèlement, la féminisation croissante de la migration sénégalaise a produit des effets au sein de la société. Le départ des femmes a été à l'origine de processus de « négociation des places » dans de nombreuses familles sénégalaises (Tandian, 2003; Sarr, 1991), tout en suscitant des perceptions socioculturelles négatives à leurs égards. Dans ces cas, la migration a souvent été associée à des pratiques « contre nature » voire « risquées » souvent considérées comme outrage aux mœurs sociétaux (Ba, 1996; Sow et Antoine, 2000 ; Rosander, 2004). 
10 En prenant en compte ces analyses anthropologiques et sociologiques, deux positionnements peuvent se formuler : en premier, la migration de l'homme et celle de la femme n'a pas la même signification au sein de la société sénégalaise. Deuxièmement, bien que l'appartenance socio-culturelle influe fortement sur les relations de couples séparés par la migration, cette influence n'est toutefois pas bénéfique aux femmes, qu'elles " partent » ou « restent ».

11 Cependant, l'approche par la géographie sociale permet d'inclure dans notre analyse, le rôle de l'acteur auquel est accordée une certaine marge de manœuvre face à un écheveau de contraintes et d'opportunités. Cette position rend possible l'appréhension des dynamiques de recompositions produites par les mariages à distance et la complexité des schémas alliant ceux qui « restent »et ceux qui «partent». Nous nous focaliserons donc ici sur les femmes mariées à des Modou-Modou ${ }^{6}$ et vivant au Sénégal et sur les migrantes qui vivent en Espagne, laissant leur conjoint et leurs enfants au Sénégal.

\section{Le poids du contexte espagnol dans les arbitrages matrimoniaux}

La Catalogne et la RMB ont offert pendant trois décennies des opportunités de vie et de travail aux Sénégalais (Niang-Ndiaye, 2014). Cependant depuis les années 2000, le renforcement et le durcissement des politiques migratoires espagnoles conjugués à une précarisation du marché du travail catalan ont changé la donne.

13 Aujourd'hui, même si la Catalogne et de manière générale l'Espagne ne pratique pas directement de « politique de quotas » en matière d'immigration, l'octroi du permis de séjour et de travail aux étrangers extracommunautaires repose sur certaines conditions (Gabrielli, 2011). Il dépend d'abord du contexte économique espagnol, mais la procédure de délivrance peut aussi varier en fonction de l'origine de la personne (Gabrielli, 2011). Même s'il est vrai que le pays a procédé à six régularisations massives de personnes étrangères depuis les années 1980 et que les procédés d'obtention d'un titre de séjour régulier sont moins draconiens que dans d'autres pays communautaires (notamment en France, et en Allemagne), le renforcement et le durcissement de la législation sur les étrangers sont devenus une réalité depuis l'entrée en vigueur de la loi Arraigo social (Loi 4/2000 du 11 janvier) ${ }^{7}$. Cette législation axée sur la personne migrante et ses caractéristiques spécifiques (revenus, statut, attaches) ne reflète pas la dimension matrimoniale, encore moins familiale de l'immigration. La complexification des mesures de regroupement familial dans le contexte espagnol influe ainsi grandement sur le choix, généralement des migrants, de faire venir leur conjointe ou pas. Les procédés de regroupement familial représentent pour nombre de migrants des dispositifs parsemés d'obstacles juridiques, économiques et sociaux. Pour faire venir sa conjointe ou son conjoint, le migrant ou la migrante doit non seulement avoir un permis de séjour régulier mais justifier de ressources économiques suffisantes prouvant sa capacité d'accueil (conditions matérielles et de logement, stabilité de l'emploi, etc.). Or, pour ceux et celles qui sont en situation irrégulière ou qui disposent d'un emploi précaire (commerçants ambulants, agriculteurs, travailleurs au noir, sans papiers etc.), le regroupement familial devient quasiment improbable. Les Modou-Modou constituent le groupe le plus concerné par cette situation. Généralement commerçants ambulants, leurs pratiques liées au travail les inscrivent souvent dans une mobilité entre différents 
espaces au gré des opportunités de vente. Dans leurs discours, hormis les contraintes réglementaires auxquelles ils doivent faire face, la réticence au regroupement familial se justifie par des choix résidentiels marqués par la colocation ou l'hébergement. Cette stratégie leur permet dès lors de dégager une épargne substantielle destinée à l'entretien des autres membres de la famille restés au pays. Au-delà du seul cadre juridique et réglementaire de la procédure de regroupement familial, cette démarche fait écho à d'autres contraintes. Dans leur calcul des avantages comparatifs en contexte migratoire, le regroupement familial n'est pas rentable et génère même des coûts supplémentaires qui supplantent de loin les envois mensuels d'argent au Sénégal. La prise en charge de la famille sur place n'exclut pas celle des autres membres de la famille au pays. Cela constitue une double charge qu'ils doivent assumer. Ensuite, l'insertion professionnelle des femmes est jugée difficile sur le marché du travail catalan. Les analyses à ce sujet attirent l'attention sur le fait que la faible participation des femmes africaines au marché du travail catalan ne peut pas être analysée uniquement en termes de position sociale dans les cultures et sociétés d'origine, mais doit aussi intégrer leur statut, notamment légal, dans la destination ainsi que l'accès à l'emploi des femmes en général, qui affichent toujours des taux d'activité et des salaires plus faibles que les hommes (Sow, 2004 ; Niang-Ndiaye, 2014). Enfin, d'autres facteurs limitant l'insertion de ces femmes sont devenus une réalité depuis la crise espagnole de 2008 : la structure du marché de l'emploi, l'accès au système de sécurité sociale ou les possibilités de garde des enfants. La plupart des mesures d'intégration à l'image des cours de langue (l'espagnol et le catalan) restent également fondées sur une approche individuelle avec parfois des horaires et obligations de fréquentation pour l'essentiel contraignantes (Niang-Ndiaye, 2014).

\section{Les femmes de "Modou-Modou », entre attentes socio-culturelles et aspirations individuelles}

Dans les années 1990, l'apparition de grandes villas et de voitures de luxe dans le paysage urbain des villes sénégalaises comme Dakar, Louga, Touba, principales pourvoyeuses de migrants, les retours de migrants au pays teintés d'ostentation et de récits idéalisés, ont été à l'origine d'un imaginaire migratoire important touchant nombre de populations frappées par la pauvreté. Partir en Europe ou se marier avec un émigré étaient devenus pour beaucoup de jeunes sénégalais et sénégalaises les principaux moyens de réussite et de diversification des revenus de la famille (Sow et Antoine, 2002 ; Tandian, 2003).

Dans la région de Louga en particulier, principale zone de départ des flux de ModouModou vers l'Espagne et l'Italie (DPS, 2000), le mariage avec un émigré a longtemps constitué un "phénomène de mode", destiné à sortir la femme de la pauvreté et à assurer à toute sa famille, un avenir meilleur. Vers les années 2000, les unions avec un Modou-Modou se sont ainsi nourries de fantasmes et de connotations ${ }^{8}$, au point de faire tache d'huile dans tout le reste du pays, de concerner autant les zones rurales que les zones urbaines, autant les femmes scolarisées que celles insérées professionnellement.

Cependant avec le temps, les problèmes suscités par ces unions se sont posés au sein de la société sénégalaise. Si de manière discursive, cette réalité est incorporée aux agendas des organisations de la société civile et aux collectifs de femmes d'émigrés, installés à travers tout le pays, elle est aussi le reflet d'une préoccupation majeure de l'opinion 
publique sénégalaise relayée par les médias nationaux. C'est ainsi qu'une grande campagne médiatique s'en est saisie depuis une dizaine d'années pour décrier les répercussions parfois néfastes de ces unions chez nombre de femmes sénégalaises. Des médias virtuels comme Seneweb.com, Leral. Net, L'Obs, ou L'Enquête (pour ne citer qu'eux), ont réalisé des reportages sur ces femmes d'émigrés à travers tout le pays, et des chaines télévisées au Sénégal se sont aussi saisies de la question. La Télévision Futurs Médias (TFM) à travers une émission dénommée Wareef souligne, par l'entremise de nombreux témoignages, les diverses problématiques issues de ces unions et qui recomposent fortement les liens de parenté au Sénégal. L'analyse critique et en profondeur de divers témoignages de ces femmes a fait ressortir le rôle crucial de la famille élargie dans les dynamiques matrimoniales des couples séparés par la migration. La famille sénégalaise, même si elle corrobore au maintien de l'unité communautaire à travers le sacre du mariage, est souvent source de pratiques « ambivalentes » qui remettent en cause la durabilité des unions «transnationales » au Sénégal. Les règles de l'endogamie, le contrôle et la pression sociale, la rétribution des envois d'argent, la cohabitation avec la belle-famille, représentent autant de revers culturels, sociaux et identitaires qui mettent en péril la vie de nombreux mariages à distance. Dans leurs témoignages, apparait également en filigrane une vie de couple niée qui renvoie à l'absence du conjoint à des moments clés de la vie familiale (baptêmes, anniversaires, fêtes religieuses, etc.), comme l'illustre le témoignage de Viviane :

(...) Actuellement, cela fait plus de 10 ans, 12 ans plus précisément, qu'il n'est pas revenu au Sénégal, car il a eu des problèmes avec ses papiers, ensuite des démêlés judiciaires avec un natif du pays où il est établi (...). À dire vrai, c'est certes difficile pour moi, mais mes enfants sont mon réconfort. Souvent, j'ai envie de claquer la porte. Ensuite, je me dis que mes enfants ne méritent pas cela, (...) Imaginez! Plus de 12 ans, pour une femme mariée qui a des enfants et sans une vie sexuelle. Il faut le vivre pour en mesurer les conséquences. Le mariage, ce n'est pas simplement des coups de téléphone et ou des appels via skype, viber, bref les réseaux sociaux. Mais, c'est le contact direct, le plaisir charnel. Ce n'est pas que je suis accro au sexe, mais ce n'est pas facile. D'ailleurs, son dernier enfant, une fille, qui est aujourd'hui très grande, ne cesse de poser des questions sur son père, surtout lors des fêtes religieuses, notamment, la tabaski. Souvent, quand elle le demande, j'ai des larmes aux yeux. Heureusement pour moi, côté finance, mon époux gère bien. Rien ne me manque, à part que des milliers de kilomètres nous séparent (Reportage réalisé par le site Leral.net, $2015^{9}$ ).

Pour cette femme comme pour tant d'autres, les échanges téléphoniques et autres technologies contribuent certes à réduire les effets de la distance, mais ne suffisent guère à combler le vide, le manque et l'absence. Pour ces femmes, le contact virtuel n'est pas le contact réel. Cette absence reste d'autant plus prégnante d'après elles que les moments de retrouvailles du couple restent régis par des temporalités spécifiques, le plus souvent non programmées et calquées sur les possibilités économiques de leur mari, son calendrier de travail et parfois même sur son bon vouloir. Cette cristallisation des rapports de couple entre un avant et un après visite est parfois très mal vécue par certaines d'entre elles. Comme beaucoup ont rarement une activité professionnelle, cette situation d'enfermement au sein du cercle familial est d'autant plus accentuée que ces femmes se plaignent de solitude, d'angoisse et de manque qui poussent 
certaines d'entre elles à l'adultère et parfois à l'infanticide en cas de grossesse contractée avec un tiers (comme le montre aussi l'article d'Aurélie Fontaine, 2010). C'est ainsi que Coumba (témoignage extrait de l'émission Wareef du 9 février 2015), déclare s'être consolée de l'absence de son mari avec un de ses cousins qui réside dans la maison familiale et c'est ce dernier qui est le père de ses deux enfants. Les témoignages révèlent aussi que beaucoup de Modou-Modou ont des enfants adultérins qu'ils se voient parfois obligés de reconnaître pour sauvegarder l'image de la famille élargie. Ces pratiques qui tendent à se banaliser dans certaines zones de forte émigration, font écho aux nombreux divorces des régions les plus urbaines à l'image de Dakar. Hormis les situations de solitude et parfois d'isolement, les problèmes peuvent survenir dans les rapports avec la belle-famille surtout quand les femmes vivent dans la maison familiale (Enquêtes personnelles, 2013). Dans nombre de cas, les femmes sont confrontées à des difficultés économiques qu'elles vivent généralement dans la solitude d'autant plus que l'entourage proche ou plus éloigné, les perçoit comme des femmes épanouies, qui ne manquent de rien (Tall et Tandian, 2010; Mondain et al, 2012). Le respect des parents guide souvent l'orientation des envois d'argent, qui sont gérés par le chef de ménage et généralement par la mère du migrant. Cette dernière prend en charge les dépenses et l'entretien de toute la maison, tout en rétribuant une somme à l'épouse ou aux épouses du migrant pour leurs dépenses propres. Il est courant de voir, dans ces cas, des situations conflictuelles apparaitre dans la distribution des fonds comme le démontre l'histoire de Mariama, divorcée d'avec un Modou-Modou depuis deux ans.

Marié depuis 1999, Mariama dit avoir habité huit années dans la maison familiale de son mari. Au cours de ces huit années, Mariama a revu quatre fois son mari et a vécu avec lui 6 mois au total. Hormis les rares fois où ce dernier revenait au Sénégal, ses années passées dans la maison familiale étaient sujettes à des conflits et disputes incessants avec sa belle-famille. Elle s'occupait quotidiennement de toutes les tâches ménagères de la maisonnée et recevait de son mari 15000 FCFA par mois, que lui remettait en main-propre sa belle-mère. Avec cet argent, elle ne pouvait guère satisfaire à ses besoins les plus élémentaires et c'est souvent sa mère qui lui donnait de l'argent pour compléter ses dépenses. Parfois, elle se plaignait auprès de son mari qui appelait sa mère et ses sœurs pour résoudre les différends. Ce qui était toujours source de conflits avec ces dernières car elles ne supportaient guère ses plaintes. Ces conflits étaient parfois si violents que Mariama partait s'installer chez ses parents, ces derniers la ramenant quelques temps après. Lasse des va et vient chez ses parents, des incompréhensions avec son mari-qui, d'après Mariama, était soumis au diktat de sa mère- et des disputes avec ses belles-sœurs, Mariama demanda le divorce à son mari, qui le lui accorda sans réserve. Quelques mois plus tard, ce dernier épousa une de ses cousines (Témoignage extrait de l'émission Wareef du 4 février, 2015).

Comme décrite précédemment, ces situations sont plus fréquentes en zone rurales, où nombre de femmes ont des revenus généralement faibles, provenant souvent de petits commerces. Elles s'occupent généralement de tous les travaux domestiques. Aussi comme dans le cas de Mariama, le cercle familial proche de la femme (parents, frères, cousins) peut constituer un « rempart financier " pour aider cette dernière à satisfaire 
ses besoins. Le témoignage d'Aissatou, révèle une autre forme de contrainte à laquelle sont confrontées les femmes de Modou-Modou.

Vivant dans un appartement acheté par son mari, Aissatou, prétend voir sa bellefamille que très rarement pour éviter les problèmes. En revanche, elle déclare que même si elle vit seule dans un appartement acheté par son mari, elle n'est point heureuse. Marié depuis cinq ans, elle n'a jamais vécu avec son mari qu'elle a rencontré sur Facebook. Après maintes tentatives en vain de le rejoindre quelques temps après leur mariage (demande de visa pour l'Espagne refusée, tentative de départ échouée auprès d'un passeur) Aissatou se résigne à attendre que son mari ait des papiers et fasse le regroupement familial. Avec la crise en Espagne, son mari qui vit des conditions difficiles en Galice ne lui envoie que 20000 FCFA, par mois. Avec cette somme, elle doit prendre en charge toutes ses dépenses, et payer les factures. Elle cherche actuellement en vain du travail (Témoignage extrait de l'émission Wareef du 5 février, 2015).

L'histoire de cette femme illustre que ces mariages à distance ne sont pas que soumis au contexte du pays de départ (à travers les rapports familiaux). Les contraintes réglementaires et économiques pèsent également de tout leur poids. La crise espagnole s'est répercutée non seulement dans la sphère familiale et plus encore, dans celle intime et privée des migrants (Enquêtes personnelles, 2012). Ici les envois de fonds, comme dans le cadre familial, même s'ils ne pallient ni au manque ni à l'absence, jouent un rôle structurant des liens matrimoniaux. Il ressort donc de ces témoignages que la précarité des ressources économiques, même si elle n'est pas la cause première des problèmes ou conflits des couples, influe grandement sur leur maintien dans le temps de la migration. Nombre d'arguments qui ressortent de la quasi-totalité des témoignages, perçoivent l'autonomie financière et résidentielle des femmes, la confiance mutuelle des conjoints, comme conditions nécessaires à la durabilité de ces couples "transnationaux » sénégalais et surtout à l'épanouissement des femmes de Modou-Modou, face à un contrôle social et un opprobre sociétal qui leurs sont en tous points défavorables.

\section{Femmes migrantes, partir et laisser sa famille au pays : l'exemple de Ndeye Oumy}

Issue d'une fratrie de 8 personnes dont trois sœurs, toutes mariées et quatre frères dont un célibataire, Ndeye Oumy, une jeune femme sénégalaise de 39 ans, s'est installée à Barcelone en 2002. Venue pour la première fois en Europe en 1990 par voie de regroupement familial, Ndeye Oumy a toujours habité à Cologne en Allemagne. Cependant, son divorce en 2001 (pour des raisons inexpliquées dans son discours), l'amène à quitter l'Allemagne et à rentrer avec ses trois enfants au Sénégal, dans la maison familiale. En 2002, elle obtient un visa pour l'Espagne et laisse ses trois enfants avec sa mère pour rejoindre sa sœur cadette à Barcelone, plus précisément à Hospitalet de Llobregat. Après trois années passées dans cette ville, Ndeye Oumy n'arrivant pas à trouver un travail stable, décide en mai 2005 de retourner au Sénégal dans la banlieue Nord de Dakar. De retour dans son quartier natal, Ndeye Oumy fait la rencontre d'un homme avec qui elle se marie 6 mois 
après. La situation financière difficile de son conjoint l'amène à retourner en Espagne en 2006, laissant à nouveau ses trois enfants avec sa mère. Après 5 mois passés à chercher du travail en vain, Ndeye Oumy s'inscrit en octobre 2006 à une formation en espagnol. Un an plus tard en janvier 2007, elle est employée « au noir » comme aide à domicile à Mongat. En 2009, après deux ans passés dans cette famille catalane, son employeur lui propose un contrat d'un an renouvelable. Avec ce contrat, elle obtient un séjour régulier d'abord de deux ans et ensuite de cinq ans. Ceci lui permet de retourner deux fois $(2009 ; 2010)$ voir sa famille au Sénégal mais aussi d'envoyer régulièrement de l'argent à son mari et à sa mère pour la subsistance de ses enfants. Au moment de l'enquête, Ndeye Oumy est installée chez ses employeurs à Mongat. Elle souhaite faire venir son mari et ses trois enfants mais n'a pas encore suffisamment de ressources économiques ni de logement pour les accueillir. Elle envoie près de la moitié de son salaire au Sénégal. Elle souhaite toutefois rentrer au Sénégal pour s'occuper de son mari et de ses enfants mais est consciente qu'elle n'a pas le choix présentement. Ndeye Oumy culpabilise aujourd'hui d'avoir laissé ses trois enfants et regrette à demi-mot de s'être mariée à distance (Enquêtes personnelles, avril 2011, Barcelone).

Comme pour Ndeye Oumy, la migration de femmes sénégalaises mariées et ayant laissé leur conjoint et enfants au Sénégal est un phénomène relativement nouveau en Espagne (Niang, 2009). Dans le contexte sénégalais, cette "nouvelle migration " pose aujourd'hui un nouveau regard sur les rapports de couple et ceux de parenté (Ba, 2008 ; Babou, 2002). Même si le départ de ces femmes mariées ne renvoie pas de manière formelle à une redéfinition des rôles au sein du couple et des relations mères-enfants, il induit néanmoins à des fléchissements, des réajustements et même des bouleversements dans les rapports matrimoniaux et même sociétaux (Babou, 2002).

Le cas de Ndeye Oumy montre ainsi une situation de paradoxe dans l'idéologie socioculturelle sénégalaise. Elle doit non seulement jouer son rôle traditionnel d'épouse et de mère mais aussi assurer la subsistance familiale culturellement attribuée à son époux. Le double rôle de Ndeye acquis par la migration, justifie ainsi les sacrifices faits à l'égard de son mari et de ses enfants pour satisfaire leurs besoins matériels et émotionnels. En revanche, dans une société patriarcale, l'absence de la femme place le mari dans une situation ambivalente, à la fois de dépendance et d'égo de chef de famille à préserver. Cette situation trouve aussi son écho dans l'appellation " maribataire » qualifiant ces hommes qui traduit un certain rejet de cette situation par la société sénégalaise et surtout par la belle-famille (Enquêtes personnelles, 2013). Les femmes sont parfois culpabilisées par le réseau familial de leur époux qui vit leur départ comme une situation «contre nature ». De plus, le départ des femmes, en les positionnant comme principale contributrice de l'économie familiale, est susceptible de remettre en cause le statut traditionnel dédié aux hommes. Même si le phénomène n'est pas encore démontré par des études approfondies poussées, il est probable que les hommes, pour reconquérir ce statut au sein de la société, soient plus enclins à être polygames. Ceci explique sans doute que nombre de femmes à l'image de Ndeye Oumy essaient d'entretenir de bonnes relations avec la belle famille, en leur envoyant des cadeaux et des biens pour raffermir les liens, de peur que celle-ci encourage leur mari à prendre une autre épouse. Le contrôle familial et social induit ainsi une pression sur ces femmes car leur éloignement physique s'oppose au rôle traditionnel d'épouse qui leur est culturellement assigné. Comme dans le cas de Ndeye Oumy, cette construction 
idéologique influe grandement sur les rapports entre conjoints, qui dans bien des cas, peuvent engendrer une fragilisation des sentiments et émotions. La famille joue donc un rôle pivot dans les liens matrimoniaux. Ces liens dépendent non seulement de la place de la femme migrante au sein du réseau familial mais aussi de la nature des relations qu'elle noue avec les autres membres de la famille élargie (famille proche et belle-famille).

Quelles que soient les situations matrimoniales rencontrées, la migration induit inéluctablement des processus de recompositions et de mutations importantes au sein des couples séparés par la distance. Si l'appartenance socio-culturelle constitue un enjeu important de ces mutations dans les rapports de genre, les enjeux spatiaux, économiques et temporels doivent également être pris en compte pour décortiquer la complexité des arbitrages matrimoniaux chez les couples «transnationaux» sénégalais.

\section{Conclusion}

Le mariage à distance constitue un défi complexe et incertain, fait de bouleversements, d'attentes, d'obstacles et parfois de souffrance pour les femmes sénégalaises. La vie à distance se heurte souvent à l'enchevêtrement de situations, de contraintes et exigences familiales où elles se trouvent particulièrement éprouvées. Les témoignages de femmes font apparaître le poids des attentes différenciées de la famille élargie dans la société sénégalaise. La législation espagnole sur les étrangers dans le cadre de la procédure de groupement familial, s'interpose à la vie transnationale des conjoints. Ainsi, un dilemme perpétuel oscillant entre le contexte d'origine et familial et celui du pays d'accueil, se pose aux arbitrages des couples sénégalais. En ce sens, la durabilité du mariage à distance impliquerait une négociation du couple pour surmonter les éventuelles tensions, contraintes socio-économiques et socioculturelles qui marquent ces espaces migratoires. Une négociation qui se traduirait autant dans une certaine manière d'être et de faire avec le contexte catalan que dans une capacité à gérer le poids des héritages socio-culturels.

\section{BIBLIOGRAPHIE}

Antoine, Philippe ; Sow, Oumar (2000) Rapports de genre et dynamiques migratoires : le cas de l'Afrique de l'Ouest, In Bozon, M. ; Locoh, T. (dir.) Rapports de genre et questions de population, Paris, INED, pp. 143-160.

$\mathrm{Ba}$, Cheikh-Omar (1996) Dynamiques migratoires et changements sociaux au sein des relations de genre et des rapports jeunes/vieux des originaires de la moyenne vallée du Fleuve, Dakar, Université Cheikh Anta Diop de Dakar, 295 p.

Th. Doct. : Géographie : Université Cheikh ana Diop : 1996. 
Baizan, Pau ; Beauchemin, Cris ; González-Ferrer, Amparo (2011) A reassessment of family reunification in Europe. The case of Senegalese couples, Paris, Ined, 26 p.(MAFE Working Paper, $\mathrm{n}^{\circ} 16$ ).

Bara-Diop, Abdoulaye (1985) La Famille wolof: tradition et changement, Paris, Karthala, 262 p. Barou, Jacques (2001) La famille à distance. Nouvelles stratégies familiales chez les immigrés d'Afrique sahélienne, Hommes et migrations, $\mathrm{n}^{\circ}$ 1232, pp. 16-25.

Caarls, Kim ; Mazzucato,Valentina (2015) La migration internationale est-elle un facteur de divorce ? Les couples ghanéens au Ghana et à l'étranger, Population, vol. 70, nº 1, pp. 135-161 [Disponible sur Internet]

Charsley, Katharine (2005) Unhappyhusbands : Masculinity and migration in transnational Pakistani marriages, Journal of the Royal Anthropological Institute, vol. 11, n 1, pp. 85-105

Dial, Fatou-Bintou (2008) Mariage et divorce à Dakar. Itinéraires féminins, Paris, Karthala-CREPOS, $200 \mathrm{p}$.

Direction de la Prévision et de la Statistique (DPS) (2000) Rapport de synthèse de la Deuxième enquête sénégalaise auprès des ménages (ESAM 2), Dakar, DPS, 262 p.

Gabrielli, Lorenzo (2011) La construction de la politique d'immigration espagnole : ambiguïtés et ambivalences à travers le cas des migrations ouest-africaines, Bordeaux, Institut d'études politiques de Bordeaux, $479 \mathrm{p}$.

Th. Doct. : Sc. Politique : Université de Bordeaux : 2011.

Glick, Jennifer (2010) Connecting complex processes : A decade of research on immigrant families, Journal of Marriage and Family, vol. 72, n³, pp. 498-515.

Grillo, Ralph ; Mazzucato, Valentina (2008) Africa<>Europe : A double engagement, Journal of Ethnic and Migration Studies, vol. 34, n 2, pp. 175-198.

Hirsch, Jennifer (2003) A Court ship after Marriage : Sexuality and Love in Mexican Transnational Families, Berkeley, University of California Press, 376 p.

Jolly, Susie ; Reeves, Hazel (2005) Gender and migration, an overview report, Sussex, Institute of Development Studies (BRIDGE).

Mahler, Sarah J (2001) Transnational relationships : The struggle to communicate across borders, Identities, vol. 7, $\mathrm{n}^{\circ}$ 4, pp. 583-619.

Mazzucato, Valentina ; Schans, Djamila ; Caarls, Kim ; Beauchemin, Cris (2015) Transnational families between Africa and Europe, International Migration Review, vol. 49, n 1, pp. 142-172.

Mondain, Nathalie (2009) Rejoindre le domicile conjugal en milieu urbain : implications sur la formation des unions et la vie de couple au Sénégal, In Amadou Sanni, M. ; Klissou, P. ; Marcoux, R. ; Tabutin, D. (dir.) Villes du Sud. Dynamiques, diversités et enjeux démographiques et sociaux, Paris, Éditions des archives contemporaines, pp. 247-272.

Mondain, Nathalie ; Randall, Sara ; Diagne, Alioune ; Elliot, Alice (2012) Les effets de l'émigration masculine sur la situation des femmes au Sénégal : renforcement ou ébranlement des équilibres traditionnels, Autrepart, $n^{\circ}$ 61, pp. 81-97.

Niang-Ndiaye, Marème (2014) Habiter en migration : Sénégalais et Gambiens dans la Région Métropolitaine de Barcelone, Montpellier, Université Paul Valéry, 559 p.

Th. Doct. : Géographie : Université Paul Valéry : 2014.

Rosander, Eva Evers (2005) Cosmopolites et locales : femmes Sénégalaises en voyage, Afrique \& histoire, vol. 4, $\mathrm{n}^{\circ} 2$, pp. 103-122. 
Sow, Fatou (1991) Le pouvoir économique des femmes dans le département de Podor. Dakar, IFAN/ UCAD.

Sow, Papa (2004) Sénégalais et Gambiens en Catalogne (Espagne), Analyse géosociologique de leurs réseaux spatiaux et sociaux, Barcelone, Université Autonome de Barcelone (Thèse de doctorat en géographie humaine, non publiée).

Tall, Serigne-Mansour (2002) L'émigration internationale sénégalaise d'hier à demain, In Diop, M.-C. (dir) Les sociétés sénégalaises entre le global et le local. Paris, Karthala/UNRISD, pp. 549-578.

Tall, Serigne-Mansour (2008) La migration internationale sénégalaise : des recrutements de maind'œuvre aux pirogues, In Diop, M.-C. (dir) Le Sénégal des migrations. Mobilités, identités et sociétés, Paris, Crepos-Karthala-Onu Habitat, pp. 37-67.

Tandian, Aly (2003) Des migrations internationales à la question identitaire : redéfinition de statuts des migrants Haalpulaar et évolution des rôles féminins dans la vallée du fleuve Sénégal, Toulouse, Université de Toulouse-le-Mirail, $423 \mathrm{p}$.

Th. Doct. : Sociologie : Université Toulouse-le-Mirail et Gaston Berger, Saint-Louis : 2003.

Tandian, Aly (2008) Des migrants sénégalais qualifiés en Italie : entre regrets et résignation, In Diop, M.-C. (dir.) Le Sénégal des migrations. Mobilités, identités et sociétés, Dakar ; Paris, Crepos ; Karthala-ONU Habitat, pp. 365-388.

Zontini, Elisabetta (2010) Transnational Families, Migration and Gender. Moroccan and Filipino Women in Bologna and Barcelona, New York, Berghahn Books, $280 \mathrm{p}$.

\section{NOTES}

1. Située au centre-est de la Catalogne, la Région Métropolitaine de Barcelone (RMB), constitue l'une des plus grandes conurbations espagnoles. Elle abrite une population de 4.392 .393 habitants répartis dans 164 municipalités en 2015. Elle représente $10 \%$ du territoire de la Catalogne et concentre plus de $70 \%$ de la population catalane ( 4.8 millions d'habitants) en 2015. Depuis le verrouillage des frontières au milieu des années 1980 de la plupart des destinations classiques migratoires européennes, la RMB est devenue une "porte d'entrée " au sein de l'espace Schengen. Cet espace a ainsi constitué un important pôle migratoire des Sénégalais à partir des années 1990. Dans cette perspective, la RMB a vu s'installer progressivement des communautés sénégalaises issues de diverses régions du pays (depuis la vague Haalpulaaren des Pëlfuladu du sud du Sénégal, aux flux de Mourides en provenance du centre du Sénégal).

2. L'importance des hommes enquêtés sur les femmes s'explique par le caractère plus ancien et important de la migration masculine des Sénégalais et des Gambiens en Espagne et particulièrement en Catalogne. Pour cette étude nous avons choisi de nous focaliser sur la migration féminine sénégalaise, qui, bien que moins forte en nombre par rapport à celle des hommes, est jugée particulièrement plus importante que celle des Gambiennes et obéit à des enjeux beaucoup plus complexes.

3. Reportage - Dans l'univers infernal des femmes de Modou-Modou, 23/06/ 2015)

4. Reportage - Au Sénégal, la solitude des femmes d'émigrés : par Aurélie Fontaine, 05/10/2010.

5. Thématique de l'émission Wareef : « SeyuModou-Modou-yi » (en langue wolof, langue nationale du Sénégal et qui signifie littéralement en français ; le mariage des Modou-Modou)

6. Le mot Modou-Modou, d'après quelques interprétations et recherches et d'après nos propres investigations auprès de migrants (entre 2010 et 2013), a été utilisé pour la première fois en France, au tout début de l'immigration africaine. À l'époque où Serigne Moustapha Bassirou était le Khalif de Touba, la plupart des familles mourides avaient donné le prénom du guide religieux à 
leur fils, Modou Moustapha. À cette époque aussi, la majeure partie des gens qui se rendait en France portait ce prénom à tel point que la plupart des Français lorsqu'ils voyaient un Sénégalais l'appelaient Modou. C'est à partir de là qu'a surgi l'appellation de Modou-Modou pour désigner tout Sénégalais venu pour une immigration économique en France ; le vocable a fait tache d'huile d'abord en Europe puis à travers le monde entier.

7. L'étranger en situation irrégulière a la possibilité de demander sa régularisation en prouvant sa situation d'intégration totale en raison d'un emploi («situación de arraigo laboral»), s'il prouve qu'il a séjourné de manière continue depuis au moins deux ans et démontre l'existence d'une relation de travail en Espagne d'au moins six mois. Par ailleurs, il existe la possibilité de demander une régularisation en invoquant une situation d'intégration totale dans la société espagnole («situación de arraigo social») du fait de vivre depuis plus de trois ans en Espagne. Dans ce cas il faut apporter un contrat de travail conclu pour une durée d'au moins un an. Il faut aussi démontrer des liens familiaux avec d'autres étrangers séjournant en Espagne ou présenter un rapport d'intégration rédigé par la Communauté Autonome du domicile. Enfin, l'intéressé peut demander ce titre exceptionnel de séjour en démontrant une situation d'intégration totale pour raisons familiales ( «situación de arraigo familiar »), en tant que parent ayant en Espagne un enfant mineur de nationalité espagnole ou en tant qu'individu ayant eu un père ou une mère née de nationalité espagnole. Dans les trois cas de figure, l'intéressé doit avoir un casier judiciaire vierge (Maud Thiry, 2016). Ley Orgánica 4/2000, de 11 de enero, sobre Derechos y Libertades de los Extranjeros en España y su Integración Social).

8. Communément appelés les Venants, les Modou-Modou selon ces représentations, permettent aux femmes, grâce au lien matrimonial, de bénéficier des $3 \mathrm{~V}$ (Venant, Villa, Voitures). Autrement dit, capter les envois pour bénéficier de revenus constants, habiter dans une grande maison, participer aux festivités et répondre aux sollicitations familiales, telle est la conception qui soustend l'appellation $3 \mathrm{~V}$.

9. https:// www.leral.net/Reportage-Dans-l-univers-infernal-des-femmes-de-ModouModou_a147800.html

\section{RÉSUMÉS}

Cette contribution s'ancre dans un débat scientifique sur les tensions qui existent entre le mariage à distance et les mutations sociales et sociétales engendrées par la migration internationale. Son originalité est d'explorer le vécu, les perceptions et pratiques de femmes mariées au prisme de la séparation et de la distance inhérentes à la migration. Basée sur l'analyse de témoignages de femmes sénégalaises issus d'enquêtes de type biographique menées à Barcelone mais aussi de l'exploitation de vidéos et de documents de presse, cette étude vise à mettre en relief l'effet des contextes de migration sur les femmes mariées sénégalaises qui « restent » (au Sénégal) et sur celles parties vivre seules à Barcelone. Autrement dit, comment les arbitrages matrimoniaux s'analysent au prisme des situations contextuelles aussi bien de l'espace catalan que du Sénégal, entendues à la fois comme réalités structurelles, socio-économiques et culturelles qui caractérisent les lieux de départ et d'arrivée? 
INDEX

Mots-clés : femmes, comportement matrimonial, mariage, relations familiales, Sénégalais Index géographique : Barcelone, Sénégal

\section{AUTEUR}

MARÈME NIANG-NDIAYE

Docteure en géographie, Chercheuse associée à l'UMR ART-DEV

rema.niang@gmail.com 